\title{
RELACIÓN ENTRE ACTITUDES Y COMPORTAMIENTOS AMBIENTALES EN ESTUDIANTES DE ENFERMERÍA
}

\author{
Doriam Esperanza Camacho Rodríguez ${ }^{1}$ \\ Nelly Esperanza Jaimes Carvajal ${ }^{2}$
}

Recibido el 30 de enero de 2015, aprobado el 6 de julio de 2015 y actualizado el 24 de mayo de 2016

DOI: 10.17151/luaz.2016.43.15

\section{RESUMEN}

Introducción: Las actitudes y los comportamientos ambientales de los estudiantes de Enfermería pueden contribuir a minimizar los factores de riesgo ambiental, a través de acciones individuales o colectivas, en la vida diaria y en la práctica profesional. Objetivo: Identificar la relación entre actitudes y comportamientos ambientales en estudiantes de Enfermería de una universidad colombiana. Método: Estudio descriptivo de corte transversal en el que participaron 190 estudiantes de Enfermería. Se analizaron las actitudes y comportamientos ambientales y su relación con las variables sociodemográficas. Se analizó la correlación estadística utilizando el coeficiente de correlación de Spearman. Resultados: El $100 \%$ de las actitudes y el $46,7 \%$ de los comportamientos fueron calificados como adecuados y se encontró relación entre entre las actitudes ambientales y edad $(\rho=0,021)$, así como entre comportamientos ambientales y edad $(\rho=0,001)$ y, comportamientos ambientales sexo ( $\rho=0,012)$. Conclusiones: Aunque la mayoría de estudiantes tiene actitudes ambientales positivas, éstas no siempre se ven reflejadas en su comportamiento, haciéndose necesario mejorar la formación de los profesionales para favorecer la disminución de la morbilidad y mortalidad por enfermedades causadas como consecuencia de factores ambientales modificables.

\section{PALABRAS CLAVE}

Actitud, comportamiento, ambiente, estudiantes de Enfermería. (Fuente: DeCS/BIREME). 


\title{
RELATIONSHIP BETWEEN ATTITUDES AND ENVIRONMENTAL BEHAVIOR IN NURSING STUDENTS
}

\begin{abstract}
Objective: To identify the relationship between environmental attitudes and behaviors in nursing students of a Colombian University. Method: A descriptive cross-sectional study in which 190 students participated through a stratified sampling semester. Environmental attitudes and behaviors and their relationship to sociodemographic variables were analyzed. The statistical correlation was analyzed using the Spearman correlation coefficient. Results: $100 \%$ of the attitudes and behaviors $46.7 \%$ were rated as adequate and relationship between environmental attitudes and age $(\rho=0.021)$ and between environmental behavior and age $(\rho=0.001)$ and between environmental behavior and sex $(\rho=0.012)$ was found. Conclusions: Although most students have positive environmental attitudes, they do not always reflected in their behavior, making it necessary to improve the training of professionals to help to decrease morbidity and mortality from diseases caused due to modifiable environmental factors.
\end{abstract}

\section{KEY WORDS}

Attitude, behavior, environment, nursing student. (Source: Mesh).

\section{INTRODUCCIÓN}

De las 102 principales enfermedades y traumatismos que cubre el Informe sobre la salud en el mundo 2004, los factores de riesgo ambiental contribuyeron a la carga de morbilidad en 85 categorías, por lo cual se calcula que en todo el mundo el $24 \%$ de la carga de morbilidad (años de vida sana perdidos) y aproximadamente el $23 \%$ de todas las defunciones (mortalidad prematura) eran atribuibles a factores ambientales (Prüss-Üstün y Corvalán, 2006).

Aun cuando no todas las enfermedades son generadas por factores de riesgo ambiental, éstos sí inciden en la calidad de vida de las personas y es una obligación del Enfermero como profesional del equipo de salud, trabajar en la comprensión del medio ambiente y su influencia en la salud, para de esta manera adoptar acciones ecosistémicas en su quehacer diario y abogar por una atención 
más integral del ser humano, que incluya el cuidado de su medio ambiente (Zamberlan et al., 2013). Cabe anotar que el papel de Enfermería en la salud ambiental se evidencia desde sus inicios, ya que Nightingale (1860) indicaba, como una de las tareas de la Enfermera, el uso adecuado de aire fresco, luz, calor, la limpieza, la tranquilidad, y la selección y administración de la dieta, todo al menor gasto de energía vital para el paciente.

La Enfermería es una profesión en la cual se educa a través de acciones de promoción de salud que permiten a la persona y la comunidad ejercer la autonomía y el empoderamiento y la reflexión crítica para un cambio de comportamiento en relación con la salud ambiental. Dichas actividades deben seguir los ejes de Promoción de la Salud que se describen en la Carta de Ottawa, lo que permite el desarrollo de habilidades personales para fortalecer el desarrollo de la acción comunitaria en una articulación colectiva y revisar la formulación de políticas públicas para la creación de entornos saludables y libres de contaminación (Pinheiro et al., 2010); de hecho, debe trabajar para proporcionar argumentos a favor de la reflexión sobre la afectación del medio ambiente (Pinheiro y Santos, 2011).

De acuerdo con la Teoría de Acción Ambiental Positiva de Emmons (1997), el comportamiento humano es producto de la integración del conocimiento ambiental, los valores ambientales, la sensibilidad y las actitudes positivas hacia el ambiente, se concreta en habilidades y procedimientos personales que reflejan el convencimiento de la pertenencia del ser humano al ambiente (Hernández y Jiménez, 2010). La acción ambiental positiva es una conducta producto del empoderamiento y del sentimiento de pertenencia al ambiente. De lo anterior, se deriva la relación que tienen las actitudes con el comportamiento y con la posibilidad de influencia y control de las conductas individuales y colectivas. Entonces, se espera que, si una persona tiene una actitud favorable hacia un determinado objeto, en este caso el ambiente, tenga mayores posibilidades de que se comporte favorablemente hacia su entorno. Por tanto, el componente conductual es uno de los elementos que se puede medir de manera más directa y el más útil para evaluar la actitud (Hernández y Jiménez, 2010).

El presente estudio buscó identificar la relación entre actitudes y comportamientos ambientales en estudiantes de Enfermería de una universidad colombiana, como referente para sugerir aportes significativos a la formación de profesionales de Enfermería con el interés de intervenir oportunamente los factores de riesgo ambiental. 


\section{MATERIALES Y MÉTODO}

Estudio descriptivo correlacional de corte transversal, en el que participaron 190 estudiantes de Enfermería de una universidad colombiana. Se calculó la muestra utilizando el survey system calculator, con 95\% de nivel de confianza. Definida la muestra se realizó un muestreo estratificado por semestre académico, teniendo en cuenta el valor porcentual de estudiantes en cada semestre.

Se utilizaron dos instrumentos que miden actitudes ambientales (Castanedo, 1995) y comportamientos ambientales (Pato, Ros y Tamayo, 2005), tomando como referente la investigación de Rivera-Jacinto y Rodríguez (2009), quienes calificaron como adecuadas las actitudes con valores iguales o superiores a 32 y los comportamientos ambientales con valores iguales o superiores a 48 , tomando como base la suma de las puntuaciones obtenidas acorde con las escalas Likert utilizadas. Los cuestionarios fueron autoadministrados en el salón de clase y su diligenciamiento tuvo una duración aproximada de 10 minutos.

Teniendo en cuenta las consideraciones éticas de la Resolución 8430 de 1993, esta investigación se considera de bajo riesgo. Los participantes firmaron un consentimiento informado previo al diligenciamiento de los cuestionarios y se les informó que los resultados de la investigación solo se utilizarían con fines académicos.

\section{RESULTADOS}

El grupo de estudiantes tenía edades entre 16 y 38 años, con una mediana de edad de 21 años y el 91,1\% fueron mujeres.

Todas las actitudes ambientales fueron calificadas como adecuadas (Tabla 1), no obstante, las que obtuvieron mayor calificación fueron: el "Incremento de la contaminación puede ser perjudicial para la salud" (89\%) y "Si existiesen más campañas de educación ambiental, la situación ambiental mejoraría” $(86,4 \%)$; en orden de importancia está una actitud que es vital para que los profesionales de Enfermería se conviertan en promotores de salud ambiental y es "Me gustaría 
informar a la gente sobre la importancia y efectos de los problemas ambientales" $(84,8 \%)$.

Tabla 1. Actitudes ambientales en estudiantes de Enfermería

\begin{tabular}{|l|c|c|}
\hline Actitudes ambientales & Porcentaje & Calificación \\
\hline $\begin{array}{l}\text { La contaminación que resulta de la producción y consumo de } \\
\text { productos modernos sería más importante que sus beneficios }\end{array}$ & $38,2 \%$ & Adecuada \\
\hline $\begin{array}{l}\text { Apoyaría a que sancionen a aquellos que originen } \\
\text { contaminación }\end{array}$ & $75,4 \%$ & Adecuada \\
\hline $\begin{array}{l}\text { Los productos alimenticios envasados deberían ser de vidrio } \\
\text { retornable }\end{array}$ & $73,3 \%$ & Adecuada \\
\hline $\begin{array}{l}\text { Si existiesen más campañas de educación ambiental, la } \\
\text { situación ambiental mejoraría }\end{array}$ & $86,4 \%$ & Adecuada \\
\hline $\begin{array}{l}\text { Participaría en programas de reciclaje y reutilización de } \\
\text { basura }\end{array}$ & $82,2 \%$ & Adecuada \\
\hline $\begin{array}{l}\text { Me gustaría informar a la gente sobre la importancia y efectos } \\
\text { de los problemas ambientales }\end{array}$ & $84,8 \%$ & Adecuada \\
\hline $\begin{array}{l}\text { Incremento de contaminación podría ser perjudicial para la } \\
\text { salud }\end{array}$ & $89,0 \%$ & Adecuada \\
\hline $\begin{array}{l}\text { Creo que el uso de químicos en agricultura y de aditivos en } \\
\text { alimentos, sería peligroso para la salud }\end{array}$ & \\
\hline
\end{tabular}

Fuente: Cuestionario sobre actitudes ambientales (Castanedo, 1995) y Cuestionario sobre comportamientos ambientales (Pato et al., 2005).

En relación con los comportamientos ambientales (Tabla 2), de los 12 comportamientos evaluados, solamente $5(46,7 \%)$ fueron calificados como adecuados y ellos son: "Cierro el grifo de agua mientras cepillo mis dientes" $(69,1 \%)$, "No arrojo papeles/desperdicios al suelo" $(56,4 \%)$, "No dejo luces encendidas innecesariamente" (55,5\%), "Evito usar desodorantes conspray" $(54,45 \%)$ y "Ayudo a mantener las calles limpias" (51,83\%). 
Tabla 2. Comportamientos ambientales en estudiantes de Enfermería

\begin{tabular}{|l|c|c|}
\hline Comportamientos ambientales & Porcentaje & Calificación \\
\hline Cierro el grifo de agua mientras cepillo mis dientes & $69,1 \%$ & Adecuado \\
\hline Participo en actividades ambientales & $22,51 \%$ & Inadecuado \\
\hline Compro o consumo productos en envases retornables & $47,12 \%$ & Inadecuado \\
\hline Hablo sobre temas ambientales & $29,32 \%$ & Inadecuado \\
\hline Evito comprar o consumir alimentos que tienen químicos & $30,37 \%$ & Inadecuado \\
\hline Evito escuchar radio/televisión con volumen alto & $45,55 \%$ & Inadecuado \\
\hline Al lavar uso poco detergente/legía & $42,93 \%$ & Inadecuado \\
\hline Ayudo a mantener las calles limpias & $51,83 \%$ & Adecuado \\
\hline No arrojo papeles/desperdicios al suelo & $56,54 \%$ & Adecuado \\
\hline Veo/escucho noticias sobre temas ambientales & $43,46 \%$ & Inadecuado \\
\hline Evito usar desodorantes con spray & $54,45 \%$ & Adecuado \\
\hline No dejo luces encendidas innecesariamente & $55,50 \%$ & Adecuado \\
\hline
\end{tabular}

Fuente: Cuestionario sobre actitudes ambientales (Castanedo, 1995) y Cuestionario sobre comportamientos ambientales (Pato et al., 2005).

Al realizar un contraste entre las actitudes en relación con los comportamientos ambientales se observa que no existe coherencia entre las actitudes y los comportamientos, ya que el $89 \%$ de los estudiantes coincide en que el incremento de la contaminación podría ser perjudicial para la salud y el $75 \%$ apoyaría que sancionen a aquellos que originen contaminación, sin embargo, solamente el $45 \%$ de los participantes evita escuchar radio o televisión con volumen alto, el $42 \%$ al lavar usa poco detergente o legía, el $51 \%$ ayuda a mantener las calles limpias, el $56 \%$ no arroja papeles o desperdicios al suelo y el 55\% no deja las luces encendidas innecesariamente. Por otra parte, el $73 \%$ coincide en que los productos alimenticios envasados deberían ser de vidrio retornable, pero el $47 \%$ compra o consume productos en envases retornables; el $84 \%$ cree que el uso de químicos en agricultura y de aditivos en alimentos, sería peligroso para la salud, pero solamente el $30 \%$ evita comprar o consumir alimentos que tienen químicos.

En relación con la información, al $84 \%$ le gustaría informar a la gente sobre la importancia y efectos de los problemas ambientales, pero solamente el $29 \%$ habla de temas ambientales y el $43 \%$ ve o escucha noticias sobre temas ambientales; el 
$86 \%$ coincide en que si existiesen más campañas de educación ambiental, la situación mejoraría, pero solamente el $22 \%$ participa en actividades ambientales.

Al analizar la correlación estadística utilizando el coeficiente de correlación de Spearman se observa relación entre las actitudes ambientales y edad $(\rho=0,021)$, así como entre comportamientos ambientales y edad $(\rho=0,001)$ y comportamientos ambientales y sexo $(\rho=0,012)$.

\section{DISCUSIÓN}

El análisis de las actitudes y comportamientos ambientales en estudiantes de Enfermería es un tema que es de interés actualmente, debido al incremento de la visibilidad de esta profesión en el área de atención primaria en salud.

En este estudio se encontró que todos los estudiantes manifiestan tener actitudes ambientales adecuadas y por ende se esperaría que los comportamientos ambientales fuesen adecuados también; no obstante, en un estudio realizado en España se encontró que el compromiso ecológico efectivo y verbal o intencional es mayor que el compromiso ecológico real (Fraj y Martínez, 2005). En un estudio realizado en Chile con estudiantes de ingenierías, se observó en algunos casos que a pesar de que los alumnos poseen una actitud ambiental positiva no realizan la conducta relacionada con la misma frecuencia, lo cual se puede explicar debido a que el comportamiento proambiental también se ve afectado por otros factores (Heyl, 2012). De acuerdo con Castanedo (1995) cuando los individuos completan cuestionarios de escalas de actitudes, éstos tienden con facilidad a dar respuestas socialmente aceptadas, por lo cual puede existir incongruencia entre lo que la persona dice que hace y lo que realmente hace, por lo cual, para disminuir el riesgo de que esto ocurriera, antes de la recolección de información se hizo énfasis a los estudiantes acerca de la importancia de contestar de forma verídica, teniendo en cuenta que la encuesta era anónima y no iba a generar comentarios directos hacia uno u otro individuo.

Cabe anotar que las actitudes ambientales son las opiniones que se tienen acerca de proteger el ambiente y conservar los recursos (López y Qiroga, 2006) y éstas influyen en los comportamientos proambientales de las personas (De Castro, 2001); de otro lado, las creencias y actitudes que posee la persona influyen en los valores y éstos a su vez afectan la forma de vida de los seres humanos y su 
entorno, es decir, el ambiente en general (Mata et al., 2003). Por su parte, Stern y Oskamp (1987) mantienen que existe una relación positiva entre las actitudes a favor del medio y la realización de conductas proambientales, mientras que Taylord y Todd (1995) entienden la actitud ambiental como un determinante directo de la predisposición hacia acciones a favor del medio (Fernández et al., 2003). Según Corral-Verdugo (2001) para valorar una actitud se debe atender a los conocimientos o creencias sobre el tema, la disposición (favorable o desfavorable) a actuar en una dirección determinada.

Es amplia la creencia de que las conductas y comportamientos ambientales de los ciudadanos (reciclado de productos, adquisición de electrodomésticos de bajo consumo energético, empleo de medios de transporte público o no contaminantes, reducido consumo energético, etc.) vienen determinados por sus actitudes hacia el medio ambiente (Martínez-Carrasco, Martínez y Gázquez, 2008). La conducta proambiental es el conjunto de acciones deliberadas y efectivas que responden a requerimientos sociales e individuales y que resultan en la protección del medio (Fernández et al., 2003) y ésta se puede inferir a partir del conocimiento de las creencias que alimentan ciertas actitudes y de la disposición a actuar en una determinada dirección (Heyl, 2012). Las conductas menos frecuentes en este estudio fueron participar en actividades ambientales (10,5\%) y hablar sobre temas ambientales con personas del entorno $(27,3 \%)$, similar a lo encontrado por RiveraJacinto y Rodríguez-Ulloa (2009) quienes además encontraron un mediano interés por ver o escuchar noticias de corte ambiental $(58,8 \%)$.

Como la gran mayoría de los problemas ambientales que existen están estrechamente relacionados con el actuar de los seres humanos, una de las formas para enfrentar este problema ha sido crear planes o programas de intervención con el objetivo de fomentar una conducta que sea más amigable con el medio ambiente y que considere los impactos provocados por las acciones de los seres humanos (Heyl, 2012). Algunos de los modelos diseñados para intentar explicar, describir y predecir la realización de conductas responsables con el medio ambiente plantean que los individuos solo realizan conductas ambientalmente responsables cuando están suficientemente informados sobre la problemática ambiental, se encuentran motivados hacia ella y, además, se ven capaces de generar cambios cualitativos, están convencidos de la efectividad de su acción y de que ésta no les generará dificultades importantes (Álvarez y Vega, 2009). En investigaciones se ha encontrado que estudiantes que tienen educación o cursos sobre el medio ambiente tienen actitudes ambientales mayores que los otros alumnos (Heyl, 2012). Al respecto, caber anotar que al revisar los planes de 
estudio de las 64 facultades de Enfermería del país (SNIES, 2015), se evidenció que no existe formación explícita en el tema de salud ambiental, sino que está inmersa dentro de la formación en salud pública.

En una investigación realizada en La Habana sobre cocimientos y actitudes de la población de un municipio en cuanto al saneamiento básico ambiental y su relación con la salud, se encontró una insuficiencia en la educación sanitaria en una parte apreciable de la población, lo que a su vez incide en la actitud de los habitantes para participar de forma activa en la solución de las deficiencias que aún existen en los servicios comunitarios de saneamiento (Quintana et al., 2000). Esto demuestra la importancia de la alfabetización científico-ambiental de la comunidad para desarrollar una cultura ecológica que permita la sostenibilidad (Álvarez y Vega, 2009). Reflexionar sobre este tema hace repensar la óptica del cuidado/trabajo de Enfermería esbozándolo en un conocimiento basado en un enfoque de ecosistema, y por lo tanto, la búsqueda de la sostenibilidad a través del diálogo, discusión y construcción del conocimiento ecológico (Zamberlan et al., 2010).

Finalmente, se encontró que las actitudes y comportamientos ambientales son más adecuados en los estudiantes de los dos últimos semestres académicos que en los primeros, similar a lo hallado por Fernández, Rodríguez y Carrasquer (2006), donde además, los conocimientos fueron mayores en los últimos semestres y los llevó a concluir que hay más preocupación ambiental al finalizar los estudios universitarios.

\section{CONCLUSIONES Y RECOMENDACIONES}

Se recomienda a las facultades de Enfermería incluir asignaturas Electivas o transversalizar la formación en Salud ambiental, teniendo en cuenta que el rol de Enfermería es importante como promotor ambiental y de esta forma contribuir a disminuir la carga de morbimortalidad por enfermedades causadas como consecuencia de factores ambientales modificables. De igual forma, es importante la apertura de líneas de investigación ambiental para generar actitudes hacia la conservación ambiental y promover el papel del enfermero en la promoción del ambiente sano como cuidado de la salud. 


\section{POTENCIAL CONFLICTO DE INTERESES}

Las autoras declaran no tener conflicto de intereses.

\section{FUENTES DE FINANCIACIÓN}

Universidad Cooperativa de Colombia Sede Bucaramanga

\section{REFERENCIAS}

- Álvarez, P. y Vega, P. (2009). Actitudes ambientales y conductas sostenibles. Implicaciones para la educación ambiental. Revista de Psicodidáctica, 14(2), 245-260.

- Castanedo, C. (1995). Escala para la evaluación de las actitudes proambientales (ESPA) de algunos alumnos universitarios. Revista Complutense de Educación, 6(2), 253-278.

- Corral-Verdugo, V. (2001). Comportamiento proambiental. Una introducción al estudio de las conductas protectoras del ambiente. Santa Cruz de Tenerife, España: RESMA.

- De Castro, R. (2001). Naturaleza y funciones de las actitudes ambientales. Revista de Estudios Psicológicos, 22(1), 11-22.

- Emmons, K.M. (1997). Perspectives on environmental action: Reflection and revision through practical experience. Journal of Environmental Education, 29, 34-44.

- Fernández, R.; Hueto, A.; Rodríguez, L. y Marcén, C. (2003). ¿Qué miden las escalas de actitudes? Análisis de un ejemplo para conocer la actitud hacia los residuos urbanos.Ecosistemas, 12(2), 1-18. 
- $\quad$ Fernández, R.; Rodríguez, L. y Carrasquer, J. (2006). El conocimiento de las actitudes ambientales: una buena base para mejorar las conductas hacia el medio ambiente. Departamento de Medio Ambiente, Gobierno de Aragón, III jornadas de educación ambiental de la comunidad autónoma de Aragón.

- Fraj, E. y Martínez, E. (2005). El nivel de conocimiento medioambiental como Factor moderador de la relación entre la actitud y el comportamiento ecológico. Investigaciones Europeas de Dirección y Economía de la Empresa, 11(1) 223-243.

- Hernández, L. y Jiménez, G. (2010). Actitudes y comportamiento ambiental del personal del área de conservación Marina isla del coco, Costa Rica. Biocenosis, 23(1), 2-13.

- Heyl, M. (2012). Actitudes y conductas ambientales de los alumnos de la escuela de ingeniería de la PUC. Tesis para optar al grado de Magíster en Ciencias de la Ingeniería. Pontificia Universidad Católica de Chile.

- López, G. y Quiroga, E. (2006). Una aproximación a la psicología ambiental. Revista Fundamentos en Humanidades, 7(1), 157-68.

- Martínez-Carrasco, F.; Martínez, J. y Gázquez, L. (2008). Actitudes y comportamientos ambientales: ¿elementos determinantes en el consumo de alimentos ecológicos? III Congreso de la Asociación Hispano-Portuguesa de Economía de los Recursos Naturales y Ambientales. Recuperado de http://www.uibcongres.org/imgdb/archivo_dpo4406.pdf

- Mata, A.; Zúñiga, C.; Brenes, O.; Carrillo, M.; Char-Pentier, C.; Hernández, L. y Zúñiga, M. (2003). Estrategias innovadoras para la formación ambiental inicial de educadores en el campo ambiental. Informe de investigación. San José, Costa Rica: CONARE.

- Nightingale, F. (1860). Notes on nursing: what it is and what it is not. London: Editorial Harrison.

- Pato, C., Ros, M. y Tamayo, A. (2005). Creencias y comportamiento ecológico: un estudio empírico en estudiantes brasileños. Medio Ambient Comport Hum, 6(1), 5-22. 
- Pinheiro, E. y Santos, M. (2011). Nursing and environmental health in schools. Acta Paulista de Enfermagem, 25(5), 666-72.

- Pinheiro, E.; Santos, M.; Da Costa, P. y Cunha, N., (2010). Educação ambiental e enfermagem: uma integração necessária. Revista Brasileira de Enfermagem, 63(5), 848-852.

- Prüss-Üstün, A. y Corvalán, C. (2006). Preventing disease through healthy environments: Towards an estimate of the environmental burden of disease. World Health Organization.

- Del Puerto Quintana, C., Concepción Rojas, M., Puerto Rodríguez, A. y Prieto Díaz, V. (2000). Conocimientos y actitud de la población en relación con el saneamiento básico ambiental. Revista Cubana de Higiene y epidemiología, 8(2): 137-144.

- $\quad$ Rivera-Jacinto, M. y Rodríguez-Ulloa, C. (2009). Actitudes y comportamientos ambientales en estudiantes de Enfermería de una Universidad pública del norte de Perú. Revista Peruana de Medicina Experimental y Salud Pública, 26(3), 338-342.

- $\quad$ Sistema Nacional de Información de la Educación Superior -SENIES-. (2015). Búsqueda de programas de Enfermería activos. Ministerio de Educación Nacional.

Recuperado dehttp://snies.mineducacion.gov.co/consultasnies/programa/buscar.jsp?control=0.982820839 0030818

- $\quad$ Stern, P. y Oskamp, S. (1987). Managing scarce environmental resources. En Stokols, D. y Altman, I. (Eds.), Handbook of environmental psychology, (Vol. 2) (pp. 1043-1088). New York: Wiley.

- Taylor, S. y Todd, P. (1995). An integrated model of waste management behavior. A test of household recycling and composting intentions. Environment and Behavior, 27(5), 603-630.

- Zamberlan, C.; Calvetti, A.; Dei, J. y Heckler, H. (2010). Calidad de vida, salud y Enfermería en la perspectiva ecosistémica. Enfermería Global, 9(3), 1-7. 
- Zamberlan, C.; Calvetti, A.; Dei, J. y Heckler, H. (2013) Ambiente, saúde e enfermagem no contexto ecossistêmico. Revista Brasileira de Enfermagem, 66(4), 603-606.

1. Magíster en Gestión de la Calidad, Seguridad y Medio Ambiente. Coordinadora de Investigación Facultad de Enfermería, Universidad Cooperativa de Colombia sede Bucaramanga. Bucaramanga, Colombia. Autor para correspondencia.doriam.camacho@campusucc.edu.co. ORCID: 0000-0003-4547718X.

2. Magíster en Enfermería. Decana Facultad de Enfermería, Universidad Cooperativa de Colombia sede Bucaramanga. Bucaramanga, Colombia.nelly.jaimes@ucc.edu.co. ORCID: 0000-0003-3167-5687

Para citar este artículo: Camacho-Rodríguez, D.E. y Jaimes-Carvajal, N.E. (2016). Relación entre actitudes y comportamientos ambientales en estudiantes de Enfermería. Revista Luna Azul, 43, 341-353. Recuperado de http://200.21.104.25/lunazul/index.php?option=com_content\&view=article\&id=204

Esta obra está bajo una Licencia de Creative Commons Reconocimiento CC BY

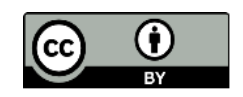

